\title{
Laparoscopic resection and Roux-en-Y reconstruction of a hilar cholangiocarcinoma
}

\author{
Jayathilake AB ${ }^{1}$, Darmapala $^{1}$, Dasanayake ${ }^{1}$, Agalawatta ASMGBB ${ }^{1}$, Madushanka UDP ${ }^{1}$, \\ Udupihilla $\mathbf{J}^{2}$, Pinto $\mathbf{V}^{3}$, Galketiya $\mathrm{KB}^{1}$ \\ ${ }^{I}$ Professorial Surgical Unit, ${ }^{2}$ Department of Radiology, ${ }^{3}$ Department of Anaesthesiology, Teaching Hospital, Peradeniya, \\ Sri Lanka. \\ Correspondence: Prof. K B Galketiya \\ e-mail: kbgalketiya@yahoo.com \\ (D) https://orcid.org/0000-0002-7464-7371 \\ Submitted on 07.09.2020 and accepted for publication on 16.01.2020
}

\section{Introduction}

Curative treatment for hilar cholangiocarcinoma is resection and Roux-en-Y reconstruction provided it is resectable and the patient being fit. Although laparoscopic resection and reconstruction is associated with less morbidity, it is a challenging surgery $(1,2)$. Laparoscopy provides a very clear picture of the anatomy by magnification and zooming which provides a huge advantage. The hybrid of experience in open resection and skills of advanced laparoscopy will allow laparoscopic approach feasible and safe (3).

A recent systematic review suggests that laparoscopic peri-hilar cholangiocarcinoma resection is still in an early phase (2). We present a case of laparoscopic excision of a Bismath type 1 hilar cholangiocarcinoma with intracorporeal biliary anastamosis and Roux-en-Y hepaticojejunostomy.

\section{Case presentation}

A 64-year-old lady presented with progressive obstructive jaundice with no history of pain. She was deeply icteric and on abdominal examination the gallbladder was not palpable. She had raised total and direct fractions of bilirubin, alkaline phosphatase and $\gamma$-glutamyl transpeptidase. Rest of her liver functions including serum albumin were normal. Her renal functions and the coagulation profile were normal.

Triple phase contrast-enhanced computerised tomography (CECT) of abdomen and pelvis showed a stricturing lesion in the common hepatic duct (CHD) extending proximally up to the confluence and distally up to the proximal part of the common bile duct (CBD) with upstream gross dilatation. There was no any hilar vessel involvement, any lymph node metastasis or any distal metastasis to lung or to the liver.

Patient did not have any co-morbidities. Preoperative percutaneous transhepatic biliary drainage was done to decompress the obstructed system as the total bilirubin level was $410 \mu \mathrm{mol} / \mathrm{L}$.

Surgical intervention was planned to follow two weeks to allow adequate decompression. Laparoscopy was decided to initially assess resectability and to proceed if resectable.

Laparoscopy was performed using five ports including the camera port (Figure 1). Gastro-hepatic ligament was dissected and hepatic artery, CBD and portal vein identified and loops placed around. The CBD was divided between ligatures and dissection continued upwards to common hepatic duct. Cystic duct and cystic artery were divided between clips and the dissection continued up to the confluence of right and left hepatic ducts (Figure 2). 


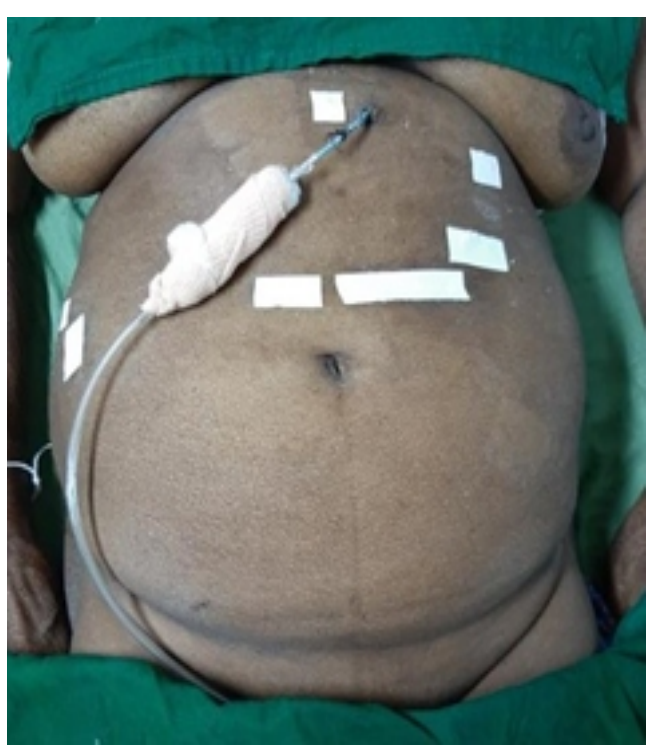

Figure 1: Post-operative view of abdomen

A jejunal loop was identified about $50 \mathrm{~cm}$ from duodeno-jejunal junction. Specimen was retrieved through the extended incision of $10 \mathrm{~mm}$ port.
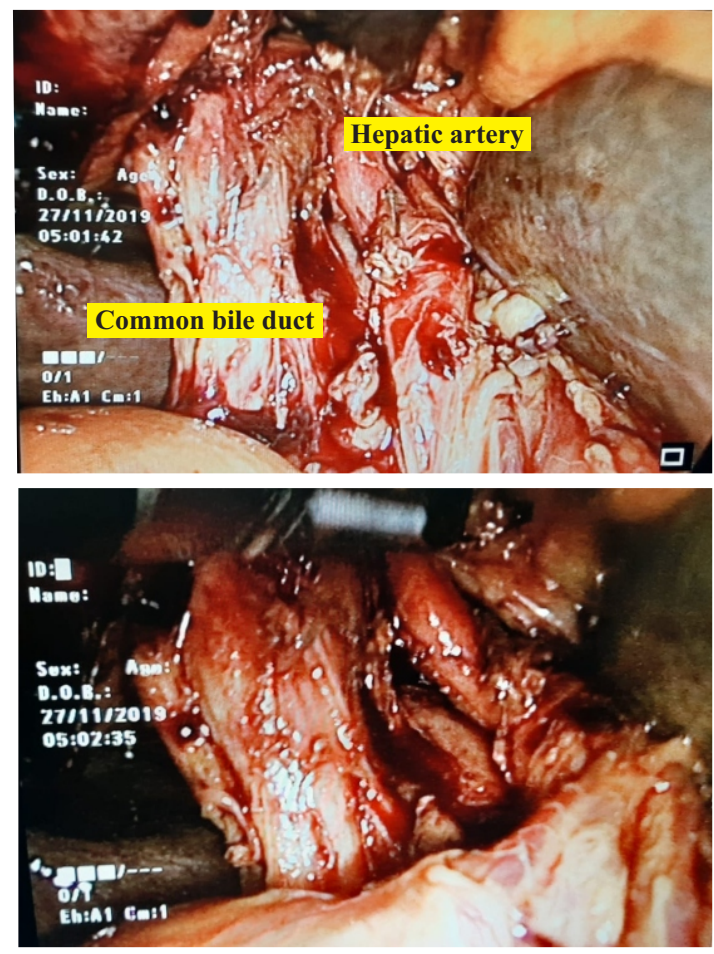

The marked jejunal loop was retrieved through the same incision and Roux-en-Y loop was created outside the peritoneal cavity. The loop was returned into the peritoneal cavity after making an enterotomy for the hepaticojejunostomy. Incision for specimen retrieval was closed using 2 polygalactin 910 sutures in two layers.

Pneumoperitoneum was recreated and end to side intracoporeal hepaticojejunostomy was done using 3-0 PDS sutures. The posterior layer was sutured with a continuous running suture. Anterior layer was by interrupted suturing.

Total operating time was $330 \mathrm{~min}$ and blood loss was roughly around $300 \mathrm{ml}$. After the procedure, a drain was inserted through one of the port sites and external biliary drain was left behind.

Post-operative ITU care was given for 4 days and patient was discharged after one week. Postoperative cholangiogram through the EBD showed no contrast leakage (Figure 3). Histopathology confirmed cholangiocarcinoma and margins were free of tumour. She was referred to an oncologist for further oncological management.
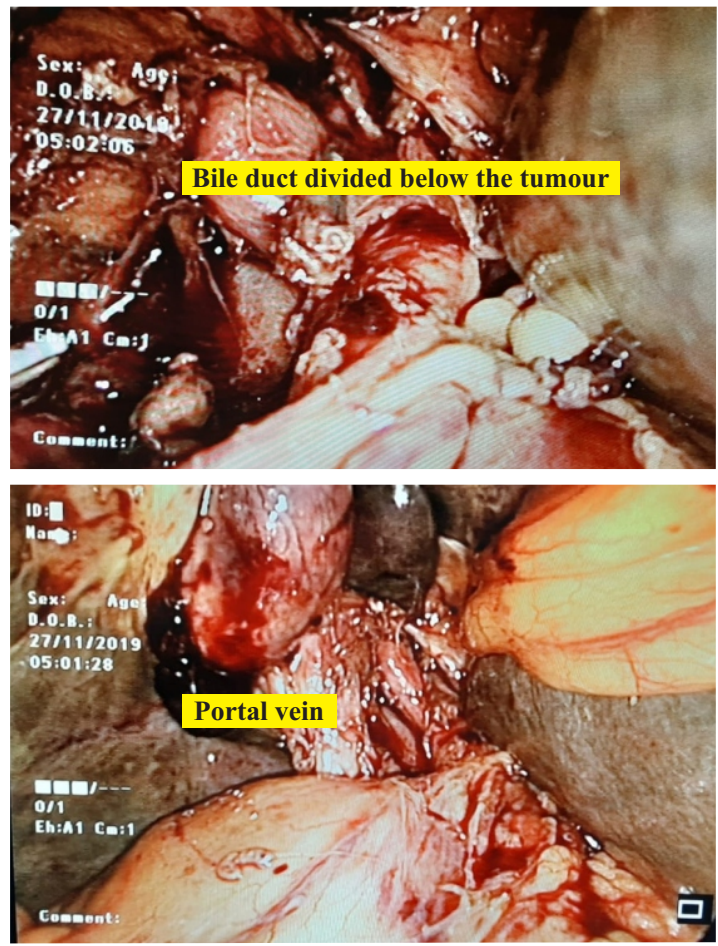

Figure 2: Images during dissection 


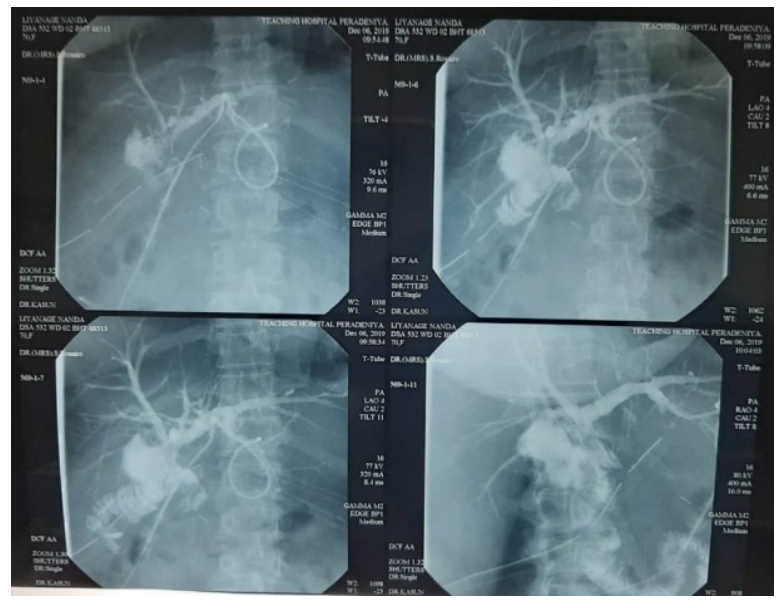

Figure 3: Post- operative cholangiogram

\section{Discussion}

Currently, only curative treatment modality for cholangiocarcinoma is surgical resection. Laparoscopic resection and intracorporeal biliary anastomosis is relatively challenging procedure (1).

However, in units with experience of open Klatskin tumour excision and advanced laparoscopic hepato-pancreatico-biliary surgery this could be performed safely (3). One of the great benefits is the clarity of the field of dissection due to magnification and zooming closer. The angle of vision too is better than in the open procedure. The gas too helps the dissection as once peritoneal folds are opened gas opens the tissue planes. At the time of hilar dissection the insufflations pressure was increased to $18-20 \mathrm{mmHg}$.

With experience, intracorporeal suturing anastomosis is easier than open surgery. This is because it is possible to zoom into, to obtain a good vision. In open surgery, as the anastomosis happens in a deep area, the technique adopted is to place a series of long interrupted sutures and then railroading. Subsequently anterior layer too is sutured with interrupted sutures. With laparoscopic suturing we found that it easier to do the posterior layer continuously. Anterior layer too could be done continuously. But we decided to do interrupted suturing as it was easier to visualise the lumina while doing interrupted suturing.
The blood loss and operative time reported in literature ranges from $300-800 \mathrm{~mL}$ and $300-600$ min respectively $(1,3)$. In this case blood loss was $300 \mathrm{~mL}$ and the operative time was $330 \mathrm{~min}$, which fall well within the above ranges. The duration of surgery is comparable to those of our open surgeries but blood loss was less. Laparoscopic resections are noted to be associated with reduced bleeding than open surgery $(1,2)$.

Another point of interest in this patient is the use of preoperative biliary decompression by a percutaneous transhepatic external biliary drainage. A recently published meta-analysis has demonstrated that preoperative internal or external biliary drainage has no significant impact on mortality or hospital stay but reduces morbidity caused by major complications (4). We prefer placing external drains instead of endoscopic placement as latter can induce inflammation which can make the dissection difficult.

\section{Conclusion}

Laparoscopic resection and intracorporeal biliary anastomosis may prove safe and feasible alternative in selected patients.

\section{References}

1. Puntambekar S, Sharma V, Kumar S, Mitkare S, Joshi G, Parikh H. Laparoscopic management of hilar cholangiocarcinoma: a case report. Indian Journal of Surgery. 2016 Feb 1; 78(1): 57-59.

2. Franken LC, van der Poel MJ, Latenstein AE, Zwart MJ, Roos E, Busch OR, Besselink MG, van Gulik TM. Minimally invasive surgery for perihilar cholangiocarcinoma: a systematic review. Journal of Robotic Surgery. 2019 May 2: 1-1.

3. Machado MA, Makdissi FF, Surjan RC, Mochizuki M. Laparoscopic resection of hilar cholangiocarcinoma. Journal of Laparoendoscopic \& Advanced Surgical Techniques. 2012 Dec 1; 22(10): 954-956.

4. Harsha Moole, Matthew Bechtold, Srinivas R Puli. Efficacy of preoperative biliary drainage in malignant obstructive jaundice: a meta-analysis and systematic review. World J Surg Oncol. 2016; 14: 82. doi: http/doi.org/10.1186/s12957-016-0933-2. 